\title{
Fuzzy Uncertainty Assessment in RBF Neural Networks using neutrosophic sets for Multiclass Classification
}

\author{
Adrian Rubio-Solis and George Panoutsos
}

\begin{abstract}
In this paper we introduce a fuzzy uncertainty assessment methodology based on Neutrosophic Sets (NS). This is achieved via the implementation of a Radial Basis Function Neural-Network (RBF-NN) for multiclass classification that is functionally equivalent to a class of Fuzzy Logic Systems (FLS). Two types of uncertainties are considered: a) fuzziness and b) ambiguity, with both uncertainty types measured in each receptive unit (RU) of the hidden layer of the RBF-NN. The use of NS assists in the quantification of the uncertainty and formation of the rulebase; the resulting RBFNN modelling structure proves to have enhanced transparency features to interpretation that enables us to understand the influence of each system parameter thorughout the parameter identification. The presented methodology is based on firstly constructing a neutrosophic set by calculating the associated fuzziness in each rule - and then use this information to train the RBF-NN; and secondly, an ambiguity measure that is defined via the truth and falsity measures related to each normalised consequence of the fuzzy rules within the RUs. In order to evaluate the individual ambiguity in the RUs and then the average ambiguity of the whole system, a neutrosophic set is constructed. Finally, the proposed methodology is tested against two case studies: a benchmark dataset problem and a real industrial case study. On both cases we demonstrate the effectiveness of the developed methodology in automatically creating uncertainty measures and utilising this new information to improve the quality of the trained model.
\end{abstract}

Index Terms-Neutrosophic sets (NS), Fuzzy Sets (FS), RBF Neural Network (RBF-NN), Receptive Unit (RU), uncertainty/indeterminacy, fuzziness, ambiguity, Charpy test Modelling.

\section{INTRODUCTION}

$\mathbf{R}$ ADIAL Basis Function Neural Networks have proved their effectiveness in several disciplines such as medicine [1], robotics [2, 3], control theory [4] image processing [5] and fuzzy modelling [6]. Furthermore, some researches and practitioners have exploited the functional equivalence established between the RBF-NN's and Fuzzy Logic Systems (FLS) [7-9] to apply the advances in fuzzy logic on the RBF-NN. Particularly, efforts on fuzzy modelling have been focused on increasing the interpretanbility and distinguishability of the rulebase while maintaining a good modelling performance in systems design [10]. For instance, in [6] a data-driven interval-type-2 neural fuzzy

Adrian Rubio-Solis is with the Department of Automatic Control and Systems Engineering, University of Sheffield, Sheffield, UK, e-mail: coq10ar@sheffield.ac.uk

George Panoutsos is with the Department of Automatic Control and Systems Engineering, University of Sheffield, Sheffield, UK, e-mail: g.panoutsos@sheffield.ac.uk

Manuscript received December 12, 2013; revised March 17, 2014. system with high learning accuracy and improved model interpretability is proposed. Juang and Chen built a type2 fuzzy model whose design is twofold: (1) an initial clustering approach was used to generate accurate fuzzy rules with good accuracy and (2) a gradient descent and ruled-ordered recursive least square algorithms for learning the antecedent and consequent parameters of the proposed network. In [11], Rhee and Choi proposed an off-line methodology based on interval type-2 fuzzy set theory for estimating the initial parameters of the RBF-NN. This work is shown to improve the classification performance and to control the linguistic uncertainty produced throughout the construction of the inference mechanism. In [12], Solis and Panoutsos proposed an RBF-NN-based neutrosophic framework for the prediction of heat treated steel properties where a neutrosophic index was designed in order to measure the inclusion uncertainty throughout the granulation process used for estimating the parameters of the RBFNN. Nevertheless, the design of logic-driven systems and interpretable models based on RUs has been an ongoing challenge in the area of modelling. While the concept of linguistic interpretability exits by default in fuzzy logic systems being established with linguistic rules and fuzzy sets associated with these rules [13], transparency is a not default property being a measure on how reliable is the linguistic interpretation of a fuzzy system. The interpretability and transparency of RBF-NN go hand in hand with the information and the highly dimensional space of data produced by the RUs. The RBF-NN posses the characteristic of fuzzy sets that the range of true value in the RUs is a closed interval of real numbers. In a like manner, the learning capabilities of the RBF-NN has some parametric flexibility that can be extended into the field of intuitionistic logic, interval type-2 fuzzy sets and neutrosophy. Particularly, neutrosophy is a generalisation of fuzzy logic [14-15] based on the fact that a proposition can be true (T), indeterminate (I) and false $(F)$, and the tuple $\langle T, F, I\rangle$ can be expressed over the real domain with no restrictions. Neutrosophy is a branch of philosophy capable of dealing with prepositions which are true and false at the same time. For this reason, in this paper we take advantage of neutrosophic set theory and some existing fuzzy uncertainty measures in order to explore and exploit the information contained and produced in each RU evaluating the role of each parameter for the system interpretation. A vast number of uncertainty measures for fuzzy sets [16-18], fuzzy relations [19] and approximate reasoning [20-21] have been proposed. 
In the design of fuzzy systems, uncertainty appears due to the lack of information, and it mainly comes into three different disguises that covers the Probabilistic Uncertainty (PU), Resolutional uncertainty (RU) and Fuzzy Uncertainty (FU). The first two types of uncertainty are closely related to belongingness of elements or events to crisp sets and the ambiguity of specifying the exact solution respectively. In this article, we propose a neutrosophic mechanism which is firstly used to measure the fuzziness I produced as a consequence of the dimensional overlapping area among RUs via defining the neutrosophic set $\langle T, F, I\rangle$, where $T$ and $F$ are the overlapping area between two RUs and its complement respectively. Secondly, an index $I$ is suggested to measure the non-specificity (ambiguity) by the RUs throughout the training stage of the RBF-NN.

The rest of this paper is organised into 3 sections: section II briefly revisits the basic theory of Neutrosophic Sets. Section III focuses in the description of the proposed fuzzy uncertainty assessment using an RBF-NN and neutrosophic sets. To measure the fuzziness and ambiguity in the course of the training process of the RBF-NN, two uncertainty indexes are suggested. In section IV, the performance of the proposed methodology is tested for modelling the well-know IRIS data set and for the identification and mechanical property prediction of the Charpy Toughness of heat-treated steel. Finally, in the concluding section the characteristics of the proposed methodology and further work are discussed.

\section{NeUtrosophic SeT}

The concept of neutrosophy was introduced by $F$. Smarandache $[14,15]$ as a generalisation of fuzzy logic, intuitionistic set, paradoxical set and paraconsistent logic in order to deal with the origin, nature and scope of neutralities. The evolution of sets from FS to NS has gone through different stages. Starting by the definition given by L. Zadeh in 1965, where a fuzzy set $A=\left\{x, \mu_{A}(x) \mid \forall x \in X, \mu_{A}(x) \in[0,1]\right\}$. Goguen defined the L-fuzzy set in $X$ as a mapping $X \rightarrow L$ such that $L^{*}, \leq_{L^{*}}$ is a complete lattice, where $L^{*}=\left\{\left(x_{1}, x_{2}\right) \in\right.$ $\left.[0,1]^{2}, x_{1}+x_{2} \leq 1\right\},\left(x_{1}, x_{2}\right) \leq L^{*}\left(y_{1}, y_{2}\right) \Longleftrightarrow x_{1} \leq y_{1}$. In 1983, Atanassanov introduced the intuitionistic fuzzy sets (IFS) as a generalisation of FS, where each element of $X$ is associated not only to its grade of membership $\mu_{A}(x) \in[0,1]$ but also to the grade of non-membership $v_{A}(x) \in[0, \overline{1}]$, but such that $\forall x \in X, \mu_{A}(x)+v_{A}(x) \leq 1$. Atanassov introduce the concept of interval-valued intuitionistic fuzzy sets (IVIFS) on a universe $X$ in 1999 as an object that $\mathrm{A}=\left\{x, M_{(A)}, N_{(A)}, x \in X\right\}$ with $M_{A}: X \rightarrow \operatorname{Int}([0,1])$ and $N_{A}: X \rightarrow \operatorname{Int}([0,1])$. IFS theory proposes an associated truth-membership function and a falsity-membership function and then such theory uses intervals was introduced as a toll to capture the uncertainty of grade of membership. Later on, Smarandache defined the neutrosophic set as a tuple $\langle T, I, F\rangle$ in the universe of discourse $X$, and the element $\bar{n} \in X$ is represented as $\bar{n}(T, I, F)$. The elements $T, I$, and $F$ are the neutrosophic logical values of a given proposition in order to deal with the associated percentage of truth $(\% T)$, the falsity
$(\% F)$ and the uncertainty/indeterminacy $(\% I)$ of an event. Neutrosophic set theory is based on infinitesimals for the definition of non-standard real-subsets $]^{-} a, b^{+}[$. A number $r$ is said to be an infinitesimal if and only if for all positive numbers $n$, and the number $r$ can be defined as $|r|<1 / n$. Where a non-standard number is defined as $-a=a-r$ and $b^{+}=b+r$. The neutrosophic tuple $\langle T, F, I\rangle$ can be evaluated by either standard or noon-standard unit intervals as follows

Let T, F and I be standard or non-standard real subsets in ]$^{-} 0,1^{+}[$with

$$
\begin{aligned}
& \sup T=t_{\text {sup }} ; \text { inf } T=t_{\text {inf }} \\
& \sup F=f_{\text {sup }} ; \quad \text { inf } F=f_{\text {inf }} \\
& \sup I=i_{\text {sup }} ; \quad \text { inf } I=i_{\text {inf }}
\end{aligned}
$$

Therefore, a neutrosophic set $\langle T, I, F\rangle$ can be interpreted as intervals, standard or non-standard real sets, discrete, continuous, single-finite sets, operations under intersection or union, fuzzy numbers, rough sets, etc.

\section{UNCERTAINTY ASSESSMENT IN RBF-NN BY USING NEUTROSOPHIC SETS}

This section presents a procedure for calculating the uncertainty during the training process of the RBF-NN. Such methodology includes two types of uncertainty assessment based on neutrosophic sets, namely: the vagueness among fuzzy rules which is estimated by calculating the fuzziness [16] between two fuzzy sets $A_{j}$ and $A_{l}$ using an overlapping coefficient [22]. And the ambiguity in fuzzy rule construction which is associated with one-to-many relations, i.e. situations with two or more alternatives during the learning process of the RBF-NN. The first step of the proposed methodology is to define the tuple $\left\langle T_{i}, F_{i}, I_{i}\right\rangle$ in the RBF-NN taxonomy and then use this information to calculate the associated uncertainty. Secondly, a process of identification must be carried out in order to estimate the RBF parameters.

\section{A. RBF-NN based on Neutrosophic Sets}

As it is mentioned in [7-8], a functional equivalence between the RBF-NN and FS can be established if the following conditions are met:

1) The number of receptive fields in the hidden layer (see Fig. 1) is equal to the number of fuzzy rules.

2) The MF's within each rule are chosen as Gaussian functions.

3) The T-norm operator used to compute each rule's firing strength is multiplication.

4) Both the RBF-NN and the FIS under consideration use the same defuzzification method, that is: either the centre of gravity or weighted sum to estimate their overall outputs.

An RBF-NN can be treated as a fuzzy inference engine that maps an input observed universe of discourse $U \subset$ $R^{n}, k=1, \ldots, n$ characterized by a MF $\mu_{A}(x): U \rightarrow[0,1]$ into the nonfuzzy $Y \in R$ set. In this paper we consider a multiinput-single-output (MISO) fuzzy system $f: U \subset R^{n} \rightarrow R$ 
which has $n$ inputs $x_{k} \in\left[x_{1}, \ldots, x_{n}\right]^{T} \in U_{1} \times U_{2} \times . . \times U_{k} . . \times$ $U_{n} \triangleq U$ where the ith rule has the form [34]

$$
\begin{gathered}
\widetilde{R}^{i}: \operatorname{IF} x_{1} \text { is } \widetilde{A}_{1}^{i} \text { and } x_{2} \text { is } \widetilde{A}_{2}^{i} \text {... and } x_{n} \text { is } \widetilde{A}_{n}^{i} \\
\text { THEN } y \text { is } \widetilde{B}^{i}
\end{gathered}
$$

where the output RU is

$$
\mu_{A^{i}}\left(\vec{x}_{p}\right)=f_{i}\left(\exp \left[-\frac{\left\|\vec{x}_{p}-\vec{x}\right\|^{2}}{\sigma_{i}^{2}}\right]\right)
$$

where $\vec{x}_{p}=\left[x_{1}, \ldots, x_{n}\right]$ and $\sigma_{i}$ and $\vec{x}$ are the width and the center of the ith fuzzy set respectively. From this perspective, the definition of a neutrosophic taxonomy using the RBF-NN can be defined as illustrated in Fig. 1. Each receptive field can be represented by the tuple $<T_{i}, F_{i}, I_{i}>$ where $T_{i}$ can be defined as the firing strength or its normalised value.

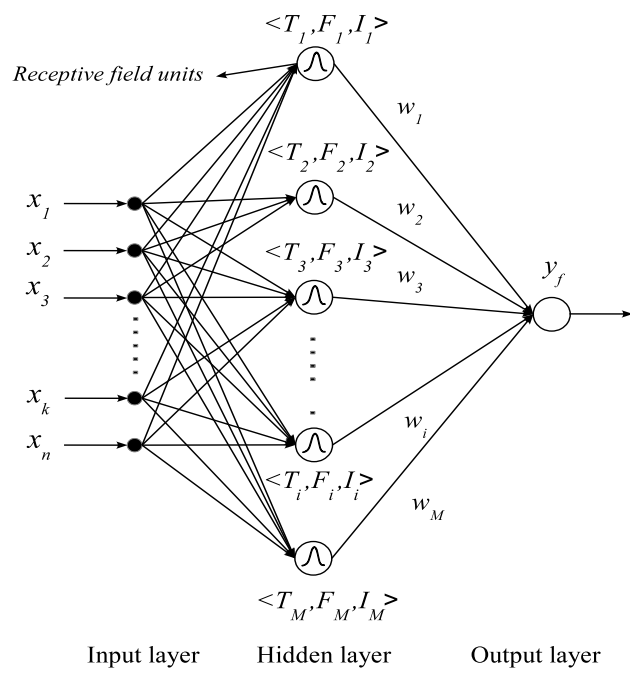

Fig. 1: RBF-NN structure based on NS

Usually, $F_{i}$ and $I_{i}$ are defined as the complement of a given fuzzy set $A_{l}$ and its associated uncertainty respectively. Therefore, the elements $T_{i}, F_{i}$ and $I_{i}$ are calculated in this paper according to fuzziness and ambiguity.

\section{B. Fuzziness}

Fuzziness or vagueness [23, 25] has been a measure widely used in the development of fuzzy set theory. Mainly, because it is associated with respect to the linguistic uncertainty of fuzzy terms. In [15] a review of a number of well known measures of fuzziness for discrete fuzzy sets is presented. In this paper we propose to use that defined in $[18,19]$ as follows:

$$
f e_{k}^{i}\left(\mu_{O v}\right)= \begin{cases}\left(1-\mu_{O v}\right)^{\alpha} e^{\mu_{O v}}+\mu_{O v}^{\alpha} e^{\left(1-\mu_{O v}\right)}, & i \neq j \\ 0, & i=j .\end{cases}
$$

Where $\alpha \in\left[0, \underline{1]}\right.$ and $\mu_{O v}$ represents the area that the fuzzy set $A_{i}$ overlaps the fuzzy set $A_{j}(j=1, \ldots, M)$ and can be obtained as:

$$
\mu_{O v}=\frac{O v_{A_{i} A_{j}}}{A_{i}}, \mu_{O v} \in[0,1]
$$

The overlapping coefficient $O v_{A_{i} A_{j}}$ is used to calculate the area under the smaller of the fuzzy distributions $A_{i}$ and

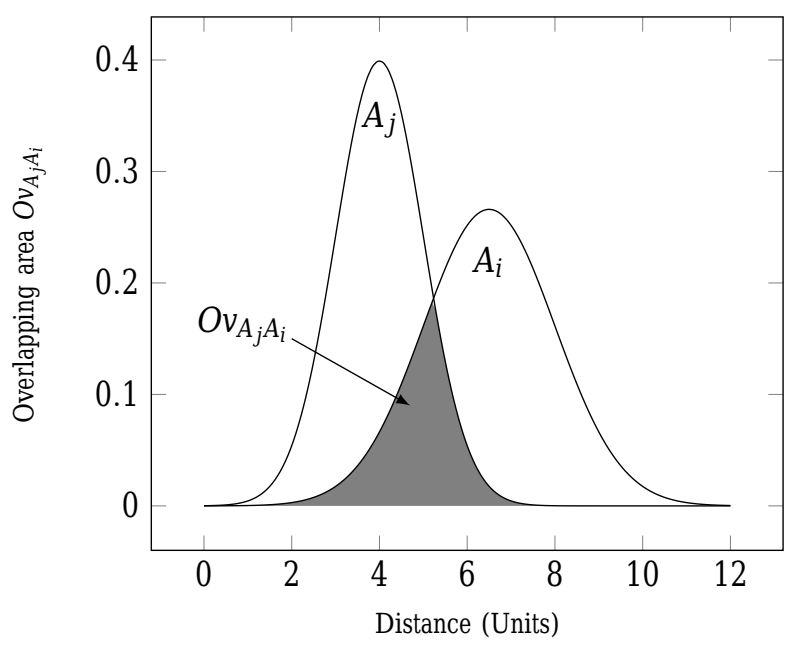

Fig. 2: Overlapping Area between the fuzzy sets $A_{j}$ and $A_{i}$

$A_{j}$ as is illustrated in Fig. 2. Therefore, $O v_{A_{i} A j}$ can be calculated as follows [22]:

$$
O v_{A_{i} A_{j}}=\int_{a}^{b} \min \left[A_{i}(x), A_{j}(x)\right] d x
$$

Eq. (2) represents the fuzziness per dimension in the ith rule between the fuzzy sets $A_{i}$ and $A_{j}$. However, the fuzziness must be an average dimensional measure per neuron at pattern $p$ which can be obtained as follows:

$$
\left.E_{i}^{p}\left(f e_{k}^{i}\right)=\frac{1}{M \times n} \sum_{k=1}^{n} \sum_{i=1, i \neq j}^{M} f e_{k}^{i}\left(\mu_{O v}\right)\right)
$$

Where $\mathrm{M}$ and $\mathrm{n}$ are the the number of rules and dimensions respectively. In order to define the neutrosophic sets based on the evaluation of the fuzziness in the fuzzy rules construction, the value of the local uncertainty/indeterminacy $I_{k}$ between two fuzzy sets $A_{i}$ and $A_{j}$ is obtained as follows:

$$
\hat{U}_{i k}^{p}= \begin{cases}\frac{1}{\left(1+e^{g \times f e_{k}^{l}},\right.}, & \mu_{O v}<\hat{t} ; \\ \frac{\left.\left(e^{g \times f e_{k}^{j}}\right)-e^{g \times f e_{k}^{i}}\right)}{\left.\left(e^{g \times f e_{k}^{l}}\right)+e^{g \times f e_{k}^{l}}\right)}, & \mu_{O v}>\hat{t} .\end{cases}
$$

When $i=j$ the value of $\hat{U}_{i k}^{p}$ is zero. Where $t \in[0,1]$ and $g \in$ $R$. Therefore the local uncertainty per RU can be defined as

$$
I_{i}=\frac{1}{M \times n} \sum_{k=1}^{n} \sum_{i=1, i \neq j}^{M} \hat{U}_{i k}^{p}
$$

And the overall network uncertainty at pattern $p$ is defined as:

$$
I_{p}=\frac{1}{M \times n} \sum_{p=1}^{P} \sum_{k=1}^{n} \sum_{i=1, i \neq j}^{M} \hat{U}_{i k}^{p}
$$

Where

$P \quad$ number of training patterns.

$T_{i}$ is defined as the truth $\mu_{A^{i}}$ associated to a receptive rule and $F_{i}=1-\mu O v$ is the falsity.

\section{Ambiguity}

Usually in fuzzy set theory ambiguity [25] includes three main types of uncertainty measures, namely: a) nonspecificity, b) dissonance and c) confusion. In this 
article, the ambiguity is associated with nonspecificity based on neutrosophic sets which represents a cognitive uncertainty. In the RBF-NN, the ambiguity is caused by the uncertainty of choosing one from all the normalized outputs (normalized firing strengths) in the hidden layer when classifying the input data. Therefore, the larger the number of alternatives, the higher the ambiguity is [25]. In this paper, the ambiguity is defined as the indeterminacy in choosing which fuzzy rule (receptive field unit) defines correctly the input data according to its normalized output. Thus, the tuple $<T_{i}, F_{i}, I_{i k}^{p}>$ is defined as follows:

The truth is calculated by:

$$
T_{i}=\frac{\mu_{A^{i}}\left(\vec{x}_{p}\right)}{\sum_{i=1}^{M} \mu_{A^{i}}\left(\vec{x}_{p}\right)}
$$

The falsity is calculated by:

$$
F_{i}=\max \left[T_{i}\right]_{i \neq j}
$$

The ambiguity/indeterminacy is obtained by using the equation defined in [27] and is depicted in Fig. 3;

$$
I_{i k}^{p}=\text { Ambiguity }_{i}=1-\left|T_{i}-F_{i}\right|
$$

Therefore, the total neural ambiguity can be calculated by the following expression

$$
I_{A}=\frac{1}{M \times n} \sum_{p=1}^{P} \sum_{k=1}^{n} \sum_{i=1}^{M} I_{i k}^{p}
$$

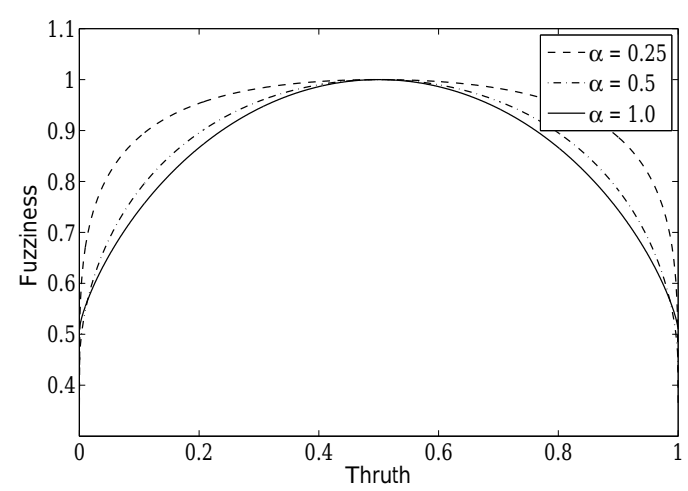

Fig. 3: Fuzziness $\left(f e_{k}^{i}\right)$

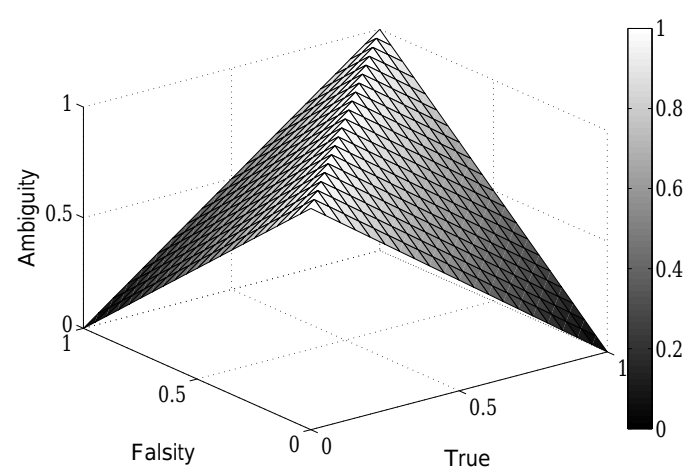

Fig. 4: Ambiguity $\left(I_{i k}^{p}\right)$

\section{Parameter identification methodology}

The parameter identification consists of two main stages: a) a process of granulation $[12,26]$ where are calculated the initial parameters of the RBF-NN and b) their corresponding optimization by using an adaptive gradient descent approach including the uncertainty from two different perspectives based on fuzziness and ambiguity. The flow diagram of the fuzzy uncertainty assessment by using RBFNN's and NS for classification is depicted in Fig. 5.

The energy expression and the objective function is obtained respectively as follows:

$$
P i=\sum_{p=1}^{P} \sum_{i=1}^{M} E_{i}^{p} e_{p}^{2}
$$

where $E_{i}^{p} e_{p}^{2}$ represents the neutrosophic inference mechanism throughout the learning process. And the fuzzy inference can be established as the weighted normalised average expressed in (11).

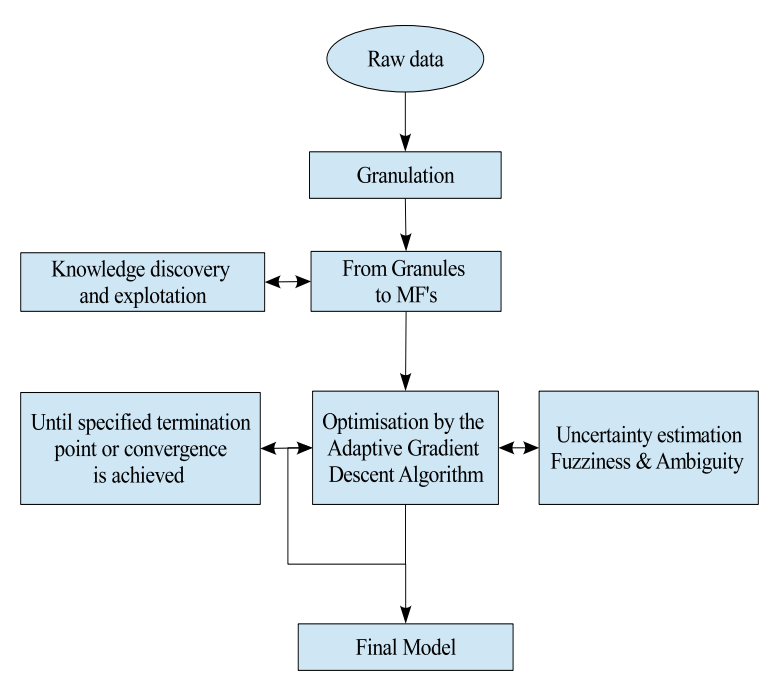

Fig. 5: Neutrosophic parameter identification process

The update rule for the output weight is:

$$
w_{i}(p+1)=\gamma w_{i}(p)-f e_{k}^{i} \beta e_{p} g_{i} ;
$$

Where $g_{i}=\frac{\mu_{A^{i}}\left(\bar{x}_{p}\right)}{\sum_{j} \mu_{A^{i}}\left(\bar{x}_{p}\right)}$ and the update rule for the width is:

$$
\sigma_{i}(p+1)=\gamma \sigma_{i}(p)-f e_{k}^{i} \beta e_{p} g_{i}\left(w_{i}(p)-y_{p}\right) \frac{\left(x_{i}(k)-C_{i k}\right)^{2}}{\sigma_{i}^{3}} ;
$$

And the update rule for the ith centre is:

$$
C_{i k}(p+1)=\gamma C_{i k}(p)-f e_{k}^{i} \beta e_{p} g_{i}\left(w_{i}(p)-y_{k}\right) \frac{\left(x_{i}(k)-C_{i k}\right)}{\sigma_{i}^{2}} ;
$$

Where $\beta$ is the learning rate and $\gamma$ is the momentum. The energy index is used to update the adaptation algorithm as follows:

- if $P i(t+1) \geq P i(t)$ Then

$$
\alpha(t+1)=h_{d} \alpha(t), \gamma(t+1)=0
$$

- if $P i(t+1)<P i(t)$ and $\left|\frac{\Delta P i}{P i(t)}\right|<\delta$ Then

$$
\alpha(t+1)=h_{i} \alpha(t), \gamma(t+1)=\gamma_{0}
$$

$$
\begin{aligned}
& \text { - if } P i(t+1)<P i(t) \text { and }\left|\frac{\Delta P i}{P i(t)}\right| \geq \delta \text { Then } \\
& \alpha(t+1)=\alpha(t), \gamma(t+1)=\gamma(t)
\end{aligned}
$$


Where $h_{d}$ and $h_{i}$ are the decreasing and increasing factors, respectively. As it is mentioned in [24], the value of the constrains are:

$$
0<h_{d}<1, h_{i}>1
$$

\section{Simulation Results}

To show the effectiveness and efficiency of the proposed methodology, two different problems of 4 and 16 dimensional space are reported here. First we explore the assessment of uncertainty due to the fuzziness by using the Iris plant database which is perhaps one of the most classic data sets in pattern recognition. The second case study under simulation is the predictive modelling of the Charpy Toughness of the Heat treated steel [12, 26]; a process that exhibits very high uncertainty in the measurements due to its thermomechanical complexity of the Charpy test itself. In this second experiment, the fuzziness and ambiguity assessment when training the RBF-NN is presented. Finally the experimental results are compared to those simulations presented in [9], [12] and [26].

\section{A. Example 1: Iris Plant Classification}

This example employs the Iris data set which contains three main categories, namely; a) Iris Setosa, b) Iris Versicolour and c) Iris Virginica of 50 instances each, where each category refers to a type of an iris plant and whose main classification feature is that one category is linearly separable from the two others and the latter are non linearly separable each other. This experiment also explores the proposed neutrosophic frameworks for creating a more distinguishable discourse of universe where RBF-NN is trained by using the $100 \%$ of data and the network uncertainty caused by the overall fuzziness related to this training process is evaluated. Table I, shows the attribute information and the summary statistics of the Iris data set and the correct percentage (\%) of average classification accuracy for the class 1,2 , and 3 by using the tuple $<T_{i}, F_{i}, I_{i}>$.

TABLE I: Iris Database statistics, attributes and average classification accuracy

\begin{tabular}{ccccc}
\hline \hline Summary Statistics & Min & Max & Mean & SD \\
\hline \hline Sepal Length $(\mathrm{cm})$ & 4.3 & 7.9 & 0.83 & 5.84 \\
Sepal Width $(\mathrm{cm})$ & 2.0 & 4.4 & 0.43 & 5.84 \\
Sepal Length $(\mathrm{cm})$ & 1.0 & 6.9 & 1.76 & 5.84 \\
Sepal Width $(\mathrm{cm})$ & 0.1 & 2.5 & 0.76 & 5.84 \\
\hline Name & class 1 \% & class 2\% & class 3 \% & \\
\hline \hline Iris & 100 & 97.66 & 99 \\
\hline \hline
\end{tabular}

In order to test the effectiveness of the proposed framework, in figure 6 the final distribution of the universe of discourse in the dimension 4 using the tuple $\left\langle T_{i}, 0,0\right\rangle$ and $\left\langle T_{i}, F_{i}, I_{i}\right\rangle$, the local uncertainty $E_{i}^{p}$ and the overall network uncertainty $I_{p}$ behaviours due to the fuzziness are illustrated respectively. Fig. 6(d), illustrates the overall fuzziness $I_{k}$ performance in the RBF network by evaluating the tuple $<T_{i}, F_{i}, I_{i}>$ throughout the training process.
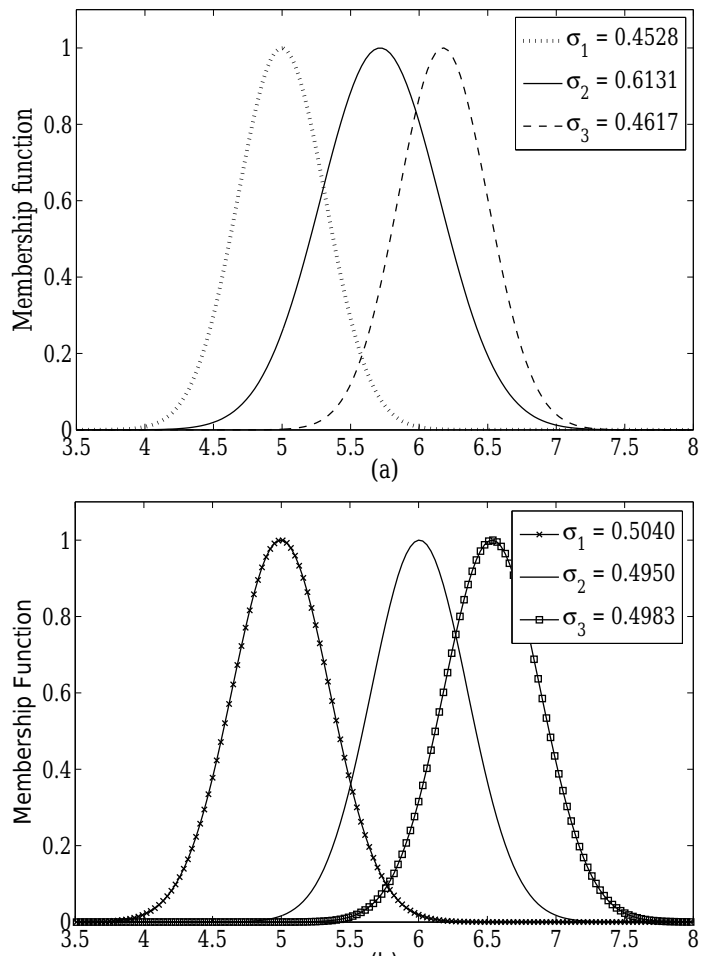

(b)

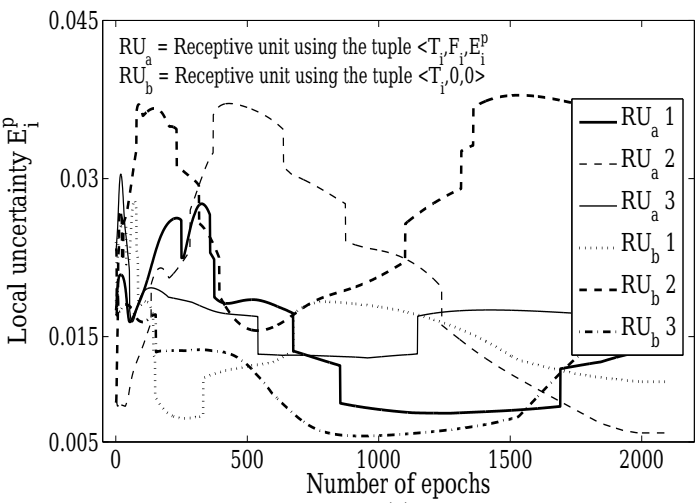

(c)

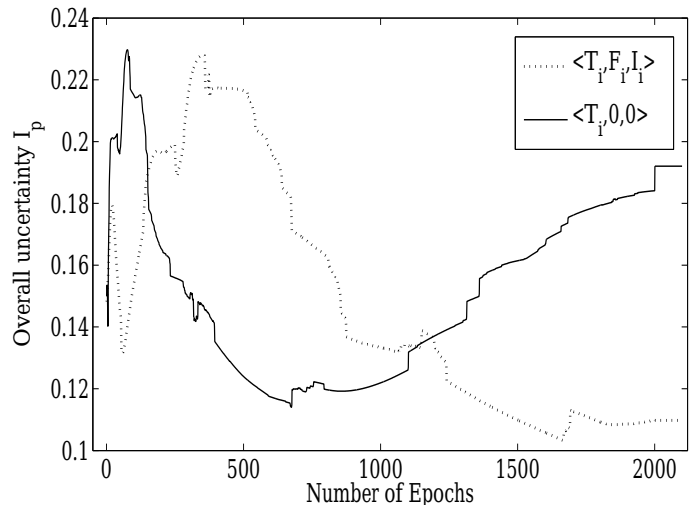

(d)

Fig. 6: (a) Final distribution using the tuple $\langle T, 0,0\rangle$, (b) Final distribution using the tuple $<T, F, I_{k}>$, (c) local uncertainty $E_{j}^{k}$ performance and (d) Overall uncertainty $I_{k}$ produced by the overlapping among the RUs throughout the training process

In Fig. 6(c), the assessment of uncertainty clearly indicates the relationship of the fuzziness and the classification of the different Iris categories. it is also obvious that for 
this case in particular, the neural network uncertainty $I_{p}$ diminished importantly when using the tuple $\left\langle T_{i}, F_{i}, I_{i}\right\rangle$ during the training. This means that it is possible to exploit the information contained in the RUs, and then manipulate the transparency and interpretability of the information per RU. The inclusion of $f e_{k}^{i}$ in this study aims to unify the concept of uncertainty and the evaluation of truth under a neutrosophic framework. In Fig. 6(d), in comparison to the overall uncertainty trend described by the RBF network by using just the tuple $\left\langle T_{i}, 0,0\right\rangle$, the overall uncertainty by using the tuple $T_{i}, F_{i}, I_{i}$ decreased sharply after the iteration 500 .

\section{B. Example 2: Charpy test}

This example is used to assess the uncertainty caused by the fuzziness and ambiguity during the training process of the RBF-NN over a real industrial case study. The example consists of a data set related to the Impact Energy Test of Heat treated grade steel. Particularly, impact energy is a highly non-linear property in relation to the steel composition, and difficult to be modelled mainly due to the multitude of standards that exists, and the variety of results that can occur under almost perfect test conditions [26]. Besides, The notorious complex results produced by the impact test can be highly scatter and low repeatable. The Charpy toughness data set used in this work consists of 1661 measurements on heat-treated steel (TATA Steel, Yorkshire, UK).

The data set has 16 input dimensions, and 1 output (Impact Energy, Joules), the scarcity of some of the data dimensions is illustrated in the Table II. For crossvalidation purposes the data have been split into training, checking and testing data sets, in order to avoid over-fitting and hence enhancing the generalisation properties when modelling the Charpy test.

The initial data used to train the RBF network consists of $1084(65 \%)$, which are composed of just raw data. The checking and testing data are 277 (17\%) and 300 (18\%) respectively. The selection of Data was set to identically match the data set used in [12] for comparison purposes. The chemical composition, test parameters and heat treatment conditions are shown in table II.

TABLE II: Charpy Toughness: Input variables

\begin{tabular}{ccc}
\hline \hline Chemical & & \\
Composition & Test Parameters & Heat Treatment \\
\hline C, Si, Mn, & Test Depth, & Hardening \\
S, Cr, Mo, & Specimen Size, & Temperature, \\
Ni, Al, V & Test Site, & Cooling medium \\
& Test Temperature & Tempering \\
& & Temperature \\
\hline \hline
\end{tabular}

In Fig. 7, a plot of the modelling results evaluating the fuzziness are illustrated. Such results are obtained by using the proposed gradient descent algorithm and the tuple $<T_{i}, F_{i}, I_{i}>$ where the term $I_{p}$ is the overall fuzziness which is computed using the Eq. (10). In Fig. 8, the final distribution by assessing the fuzziness of the fuzzy sets at dimension 3 (Test site test parameter) and the local uncertainty $E_{i}^{p}$ are illustrated. Fig. 8(b) illustrates the behaviour of the overlapping of the entire RBF-NN throughout the training process. As it is illustrated in Fig. 8(a), the higher the overlapping per dimension, the larger the local uncertainty per receptive unit (see Fig. 8(b)).

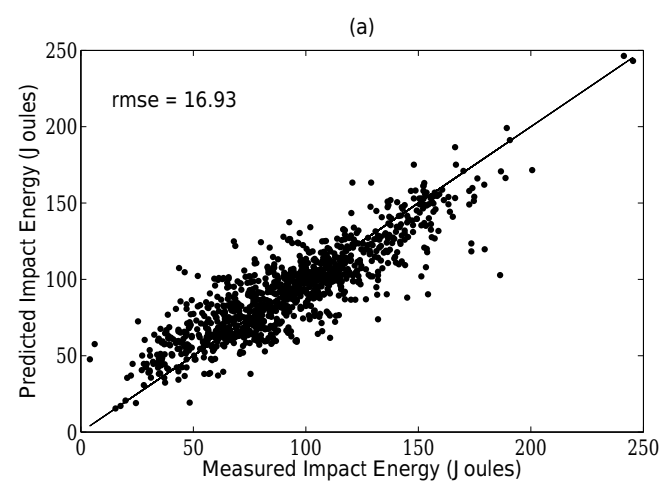

(b)

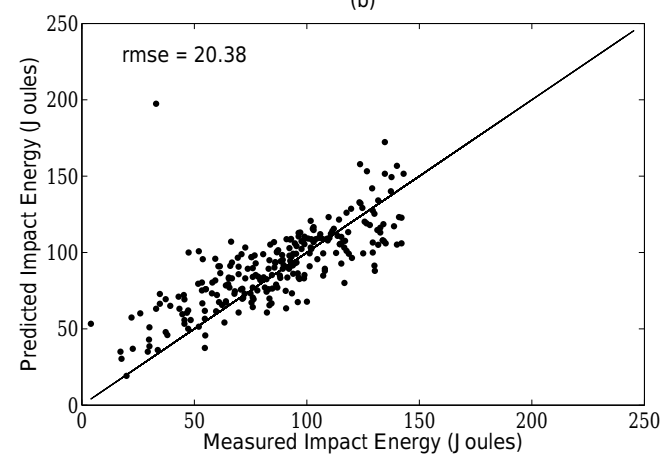

(c)

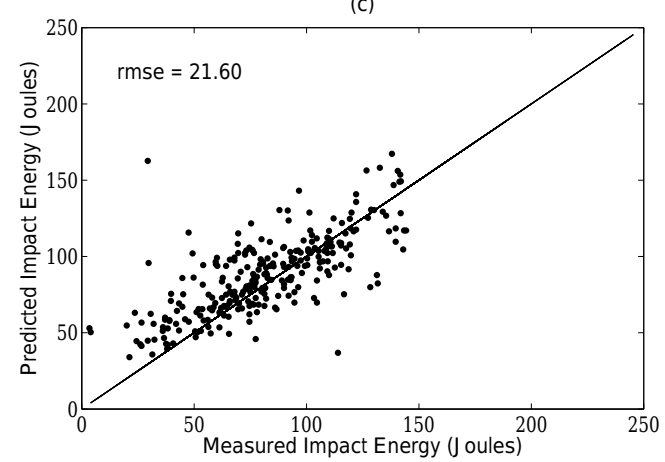

Fig. 7: Performance of (a) Training, (b) Checking and (c) Testing using the tuple $<T, F, I_{k}>$

In this sense we offer the comment that an RBF network shares the capability of fuzzy systems for dealing with situations where set-boundaries are not sharply defined [16] and the proposed fuzziness measure of the final distribution per RU contributes to the interpretability of the RBF-NN. To investigate the RBF-NN performance based on the ambiguity assessment, we then implement the proposed adaptive gradient descent algorithm [26] using the term $I_{i k}^{p}$ in the energy equation (13) instead of the term $f e_{k}^{i}$.

In Fig. 9, a plot of the simulation results is presented. Such results are comparable to those obtained by evaluating the overall fuzziness and to the RBF-NN of Mamdani type presented in [12] and [26]. The overall ambiguity index $I_{A}$ is the average ambiguity of the $\mathrm{M}$ normalised output of the RUs. Even though, Fig. 9(d) shows that the overall ambiguity behaviour over the 
span of the training process posses a decreasing trend, and the use of a measure based on ambiguity enhanced the training performance as presented in table III, the final ambiguity value is never zero. This is mainly due to high non-linear property of the steel composition and heat treatment regime. Moreover, some outliers points are equally misclassified in either by evaluating the overall fuzziness or by evaluating the overall ambiguity.

(a)

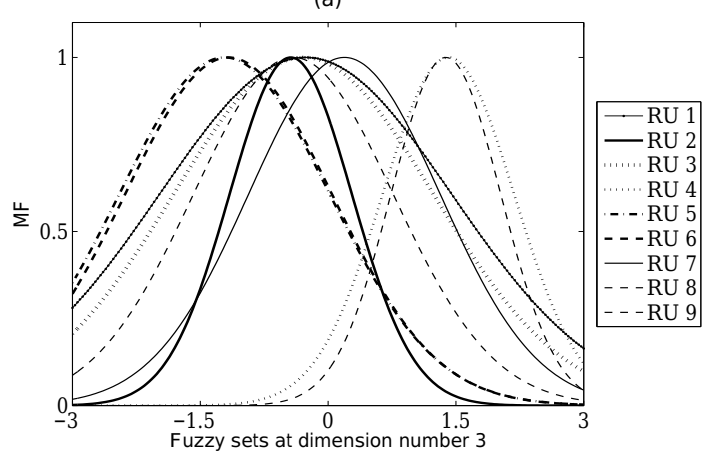

(b)

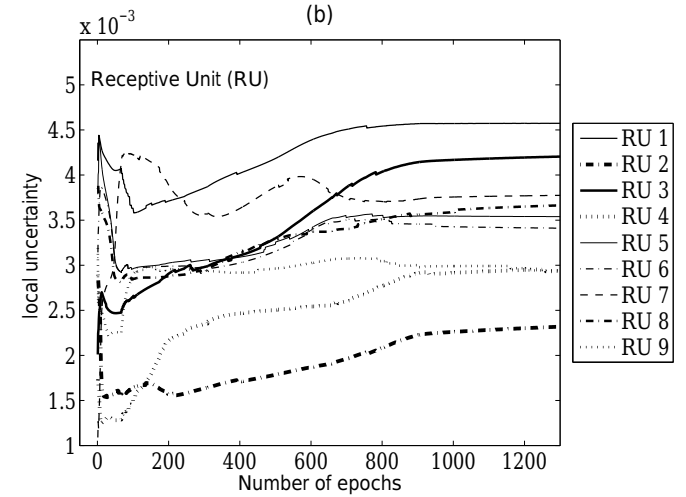

Fig. 8: (a) Final distribution using the tuple $\langle T, 0,0\rangle$, (b) local uncertainty based on fuzziness

Finally, in order to reveal that RBF-NN based on the uncertainty assessment has good performance for modelling high-dimensional problems, table III shows a comparison between three different types of uncertainty assessment, namely: using a) the tuple $\left\langle T_{i}, 0,0\right\rangle$, b) the tuple $<$ $T_{i}, F_{i}, I_{i}>$ and c) the tuple $<T_{i}, F_{i}, I_{i k}^{p}>$ which is the RBFNN of Mamdani type. As it is described in [25], in certain cases where some data were wrongly predicted mainly during the checking and testing stages; it can be concluded that such misclassification is a consequence of process repeatability of the data set (Charpy test experiments) which turns out in noisy data (or wrong data and outliers).

TABLE III: Performance of optimised RBF-NN for modelling the Charpy test.

\begin{tabular}{ccccc}
\hline \hline Model & $\begin{array}{c}\text { Number of } \\
\text { rules }\end{array}$ & $\begin{array}{c}\text { rmse } \\
\text { Training }\end{array}$ & $\begin{array}{c}\text { rmse } \\
\text { Checking }\end{array}$ & $\begin{array}{c}\text { rmse } \\
\text { Testing }\end{array}$ \\
\hline & 9 & 16.76 & 19.25 & 20.91 \\
$\langle T, 0,0\rangle$ & 9 & 16.93 & 20.38 & 21.60 \\
$\left\langle T, F, I_{k}\right\rangle$ & 9 & 16.66 & 20.25 & 21.39 \\
$\left\langle T, F, I_{i k}^{p}\right\rangle$ & 9 & & & \\
\hline
\end{tabular}

Particularly, the nature of the Charpy test produces very high data scatter and due to its low repeatability in obtaining the same results under the same input conditions, the performance of the RBF-NN is affected. In the view of the former results, the use of neutrosophic sets is not only the generalisation of fuzzy sets but also such sets can be exploited in order to increase the transparency and interpretability of systems functionally equivalence to fuzzy and then neutrosophic frameworks.

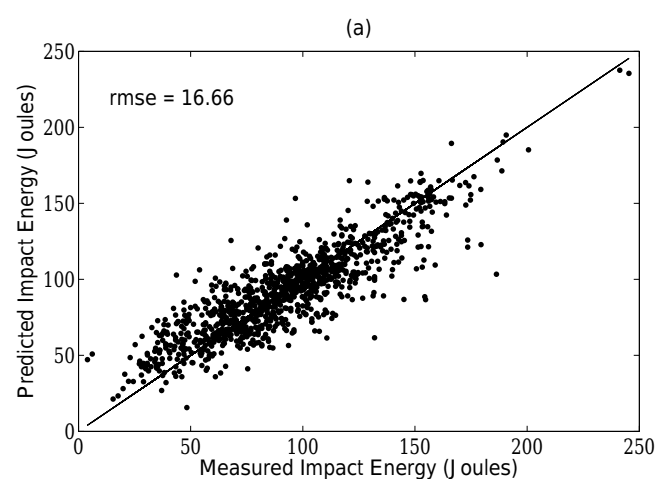

(b)
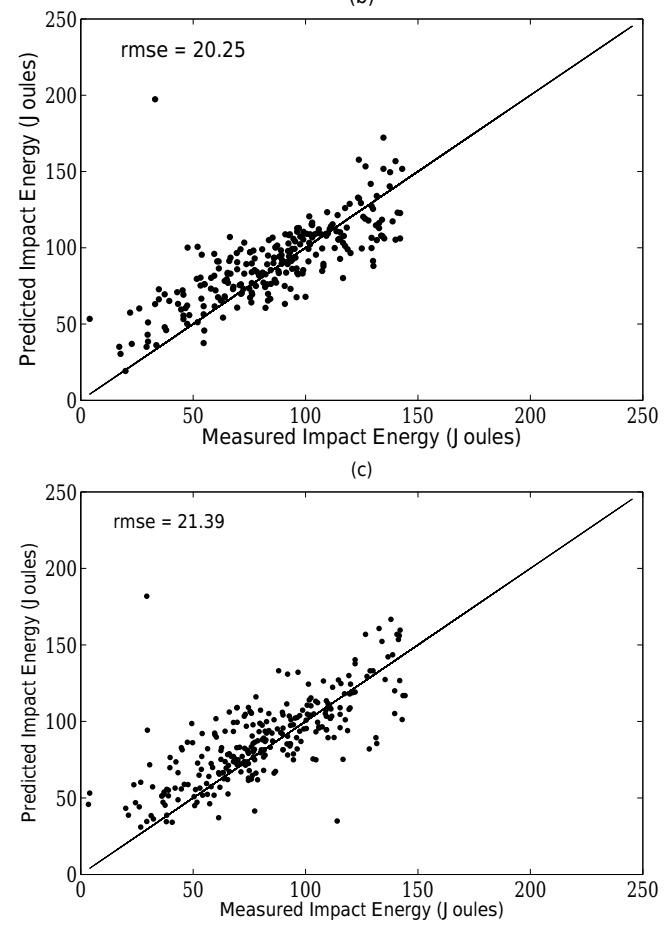

(d)

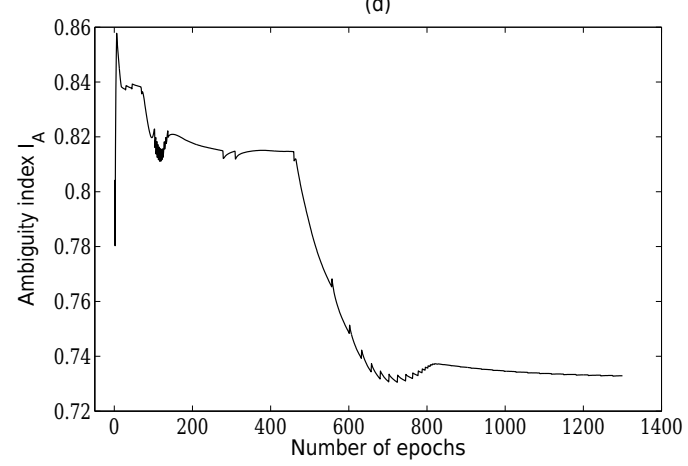

Fig. 9: Performance of (a) Training, (b) Checking and (c) Testing using the tuple $\left\langle T, F, I_{A}\right\rangle$ and (d) the behaviour of the overall ambiguity $I_{A}$ 


\section{Conclusions}

By exploiting the functional equivalence between RBFNNs and fuzzy systems of type-1, and the application of neutrosophic sets theory, we show how one may exploit the information contained in each receptive unit in an RBF$\mathrm{NN}$ to measure uncertainty and use this information to improve the modelling structure. Two uncertainty measures were considered: a) fuzziness and b) ambiguity. Firstly, we defined a fuzziness measure to examine the agreement between fuzzy rules (Gaussian fuzzy rules) by using an overlapping coefficient. Secondly, an ambiguity index was constructed based on the associated truth and falsity of each fuzzy rule, as calculated within each RU. Finally, an adaptive Back Error Propagation approach - taking advantage of the neutrosophic sets based on fuzziness and ambiguity - was employed for the parametric optimisation of the model. The presented methodology was tested against a benchmark data set and real industrial case study of high dimensionality and complex nature. The resulting modelling structures produced comparable performance to that obtained by just using fuzzy sets of type-1 (RBFNN), however this was achieved with a much simpler and more interpretable rulebase that can be further interrogated by process experts. The simplicity of the resulting structure also adds to the computational efficiency of the model, thus enabling it to be used in real-time critical applications.

\section{REFERENCES}

[1] Y. Chen, Y. Wang and B. Yang, "Evolving hierarchical RBF neural networks for breast cancer detection." 13th International Conference on Neural Information Processing, ICONIP, Hong kong, China,vol. 4234, pp. 137-144, 2006.

[2] Gao Guoqin, Ding Qinqin and Wang Wei, "Sliding mode control of parallel robot by optimizing switching gain based on RBF neural network," 31st Chinese Control Conference, pp. 975-980, 2012.

[3] Xiuli Guo, Huaimin Lu and Danfeng Du "Research and application of rbf neural network in cone picking robot," International Conference in Mechatronics and Automation, Changchun, China, pp. 1401-1405, 2009.

[4] R. Dechao, C. Xiaoqian, Y. Weiwei and C. Lu, "Small satellite attitude control based on variable structure control and RBF network," 31st Chinese Control Conference, pp. 3203-3206, 2012.

[5] Xun Cai, K. Tyagi and M.T. Manry, "An optimal construction of second order RBF neural network for approximation and illumination invariant image segmentation," The 2011 International Joint Conference on Neural Networks, pp. 3120-3126, 2011.

[6] , C. F. Juang and C. Y. Chen"Data-driven interval type-2 neural fuzzy system with high learning accuracy and improved model interpretability," IEEE Transactions on Cybernetics, vol. 43, no. 6, pp. 1781-1795, 2013.

[7] J.-S. Roger Jang and C.-T. Sun, "Functional equivalence between radial basis function neural networks and fuzzy inference systems," IEEE Transactions on Neural Networks, vol. 4, pp. 156-158, 1993.

[8] H.C. Andersen, A. Lotfi and Westphal "Comments on functional equivalence between radial basis function networks and fuzzy inference systems," IEEE Transactions on Neural Networks, vol. 9, no. 6, pp. 1529-1532, 1998.

[9] Adrian Rubio-Solis and George Panoutsos, "Interval Type-2 Radial Basis Function Neural Network: a modelling framework," IEEE Transactions on Fuzzy Systems, In press, 2014.

[10] S.-M. Zhou and J. Q. Gan, "Low-level interpretability and high-level interpretability: a unified view of data-driven interpretable fuzzy system modelling," Fuzzy Sets and Systems, vol. 159, pp. 3091$3131,2008$.

[11] F. C.-H. Rhee and B.-I. Choi, "Interval type-2 membership function design and its application to radial basis function neural networks," IEEE Int. Conference on Fuzzy Systems, vol. 6, pp. 1-6, 2007.

[12] A.R. Solis and George Panoutsos, "Granular computing neuralfuzzy modelling: a neutrosophic approach," Applied Soft Computing, Elsevier, vol. 13, no. 9, pp. 4010-4021, 2013.
[13] Andri Riid and Ennu Rüstern, "Transparent in fuzzy systems in modelling and control," Studies in Fuzziness and Soft Computing, Springer, vol. 128, pp. 452-476, 2003.

[14] F. Smarandache, "Definition of neutrosophic logic, a generalization of the intuitionistic fuzzy logic, in: Proceeding of the third conference of the European Society for fuzzy logic and technology," 2003.

[15] F. Smarandache, "A unifying field in logics: neutrosophic logic, neutrosophy, neutrosophic set, neutrosophic probability and statistics," American Research Press, 1998.

[16] N. R. Pal and J. C. Bezdek, "Measuring fuzzy uncertainty," IEEE Transactions on Fuzzy Systems, vol. 2, no. 2, pp. 107-118, 1994.

[17] Ronald R. Yager, "Uncertainty representation using fuzzy measures," IEEE Transactions on Systems and Cybernetics, vol. 32, no. 1, pp. 13-20, 2002.

[18] H. Xiaoshu and X. Fanlun, "Measures of fuzziness and entropy of fuzzy information," Proceedings of the 3rd World Congress on Intelligent Control and Automation, vol. 4, pp. 2448-2452, 2000.

[19] D. Yu, Q. Hu and C. Wu, "Uncertainty measures for fuzzy relations and their applications," Applied Soft Computing, vol. 7, pp. 1135$1143,2007$.

[20] D. Dubois and H. Prade, "Fuzzy sets in approximate reasoning, Part 1: Inference with possibility distributions," Fuzzy Sets and Systems, vol. 40, pp. 143-202, 1991.

[21] D. Dubois, J. Lang and H. Prade, "Fuzzy sets in approximate reasoning, Part 2: logical approaches," Fuzzy Sets and Systems, vol. 40, pp. 203-244, 1991.

[22] H.F. Inman and E.L. Bradley, "The overlapping coefficient as a measure of agreement between probability distributions and point estimation of the overlap of two normal densities," Communications in Statistics-Theory and Methods, Francis \& Taylor, vol. 18, no. 10, pp. 3851-3874, 1989.

[23] U. Hohle, "Entropy with respect to plausability measures," Proc. 12th IEEE Int. Symp. Multiple Valued Logic, pp. 167-169, 1982.

[24] N. R. Pal and S. K. Pal, "Object-Background segmentation using new definitions of entropy," IEE Proc. , vol. 136, pt. E, pp. 284-295, 1989.

[25] Xi-Zhao, Ling-Cai Dong and Jian-Hui Yan, "Maximum ambiguitybased sample selection in fuzzy decision tree induction," IEEE Transactions on Knowledge and Data Engineering, vol. 24, no. 8, pp. 1491-1505, 2012.

[26] G. Panoutsos and M. Mahfouf, "A Neural-Fuzzy framework based on Granular Computing: Concepts and Applications," Fuzzy Sets and Systems, vol. 161, no. 21, pp. 2808-2830, 2010.

[27] P. Kraipeerapun, "Binary classification using ensemble neural networks and interval neutrosophic sets," Neurocomputing, vol. 72, no. 13-15, pp. 2845-2856, 2009. 